\title{
Erratum to: Pronounced genetic structure and low genetic diversity in European red-billed chough (Pyrrhocorax pyrrhocorax) populations
}

\author{
Marius A. Wenzel • Lucy M. I. Webster • Guillermo Blanco • \\ Malcolm D. Burgess • Christian Kerbiriou • Gernot Segelbacher • \\ Stuart B. Piertney $\cdot$ Jane M. Reid
}

Published online: 5 December 2014

(C) Springer Science+Business Media Dordrecht 2014

\section{Erratum to: Conserv Genet (2012) 13:1213-1230 \\ DOI 10.1007/s10592-012-0366-6}

In the original publication, Tables 3 and 6 were published with incorrect estimates of population heterozygosities. All other diversity statistics were correct as originally presented. Updated versions of Tables 3 and 6 with corrected heterozygosity estimates confirmed using Arlequin 3.5 (Excoffier and Lischer 2010) as in Dávila et al. (2014) are provided in this erratum. Discrepancies were minor for populations on the British Isles. The correct estimates for
Spain are slightly larger than those reported for La Palma by Dávila et al. (2014), but this does not necessarily affect their interpretation that choughs on La Palma may have originated from multiple migration events. The original conclusion that chough populations on the British Isles have low genetic diversity compared to continental European populations remains and is now, in fact, strengthened.

The online version of the original article can be found under doi:10.1007/s10592-012-0366-6.

\footnotetext{
M. A. Wenzel · L. M. I. Webster · S. B. Piertney ·

J. M. Reid ( $\square)$

Institute of Biological and Environmental Sciences, University

of Aberdeen, Zoology Building, Tillydrone Avenue,

Aberdeen AB24 2TZ, UK

e-mail: jane.reid@abdn.ac.uk

G. Blanco

Department of Evolutionary Ecology, National Museum of Natural History (MNCN-CSIC), c/ José Gutiérrez Abascal 2, 28006 Madrid, Spain

M. D. Burgess

Centre for Research in Animal Behaviour, College of Life \& Environmental Sciences, University of Exeter,

Exeter EX4 4QG, UK

C. Kerbiriou

Muséum National d'Histoire Naturelle CERSP UMR 7204

MNHN-CNRS-UPMC, 61 rue Buffon, 75005 Paris, France

G. Segelbacher

Department of Wildlife Ecology and Management, University

of Freiburg, Tennenbacher Str. 4, 79106 Freiburg, Germany
} 
Table 3 Genetic diversity statistics (means \pm 1 SD) derived from 16 microsatellite loci across 348 red-billed choughs from 11 populations

\begin{tabular}{lcclllllll}
\hline Population & $n$ & Missing data $(\%)$ & $n_{\mathrm{a}}$ & $a_{\mathrm{r}}$ & $n_{\mathrm{e}}$ & $n_{\mathrm{p}}$ & $H_{\mathrm{O}}$ & $H_{\mathrm{E}}$ \\
\hline Colonsay & 40 & $4.85 \pm 9.01$ & $2.88 \pm 1.15$ & $1.95 \pm 0.58$ & $1.60 \pm 0.52$ & 1 & $0.28 \pm 0.19$ & $0.32 \pm 0.20$ & $0.131^{*}$ \\
Islay & 77 & $6.51 \pm 10.63$ & $3.13 \pm 1.31$ & $1.97 \pm 0.67$ & $1.62 \pm 0.62$ & 2 & $0.31 \pm 0.22$ & $0.31 \pm 0.22$ & 0.024 \\
Isle of Man & 41 & $5.95 \pm 10.85$ & $3.13 \pm 1.50$ & $1.98 \pm 0.67$ & $1.63 \pm 0.55$ & 4 & $0.32 \pm 0.22$ & $0.32 \pm 0.22$ & 0.019 \\
Ireland & 26 & $6.31 \pm 10.30$ & $3.63 \pm 1.50$ & $2.53 \pm 0.89$ & $2.16 \pm 0.95$ & 1 & $0.41 \pm 0.21$ & $0.46 \pm 0.22$ & $0.130^{*}$ \\
North Wales & 73 & $7.18 \pm 12.48$ & $3.38 \pm 1.54$ & $2.46 \pm 0.79$ & $2.29 \pm 0.83$ & 1 & $0.50 \pm 0.26$ & $0.49 \pm 0.24$ & -0.009 \\
South Wales & 11 & $6.55 \pm 6.27$ & $2.69 \pm 1.01$ & $2.34 \pm 0.70$ & $2.03 \pm 0.63$ & 0 & $0.51 \pm 0.27$ & $0.45 \pm 0.21$ & -0.078 \\
Cornwall & 9 & $19.78 \pm 25.04$ & $2.25 \pm 0.77$ & $2.05 \pm 0.62$ & $1.75 \pm 0.55$ & 0 & $0.41 \pm 0.26$ & $0.37 \pm 0.21$ & -0.031 \\
Brittany & 18 & $3.67 \pm 4.67$ & $2.81 \pm 1.17$ & $2.23 \pm 0.70$ & $1.96 \pm 0.74$ & 1 & $0.45 \pm 0.25$ & $0.42 \pm 0.22$ & -0.040 \\
French Alps & 14 & $13.07 \pm 18.43$ & $4.88 \pm 1.67$ & $3.55 \pm 0.86$ & $3.33 \pm 1.30$ & 5 & $0.58 \pm 0.16$ & $0.66 \pm 0.12$ & $0.167 * *$ \\
Spain & 17 & $4.35 \pm 10.22$ & $6.38 \pm 2.55$ & $4.11 \pm 1.17$ & $4.51 \pm 1.92$ & 28 & $0.77 \pm 0.18$ & $0.73 \pm 0.15$ & -0.038 \\
Paradise Park & 22 & $3.86 \pm 8.77$ & $2.81 \pm 1.05$ & $2.38 \pm 0.67$ & $2.15 \pm 0.61$ & 0 & $0.53 \pm 0.23$ & $0.48 \pm 0.20$ & -0.063 \\
Total & 348 & $6.57 \pm 11.58$ & - & - & - & - & - & - \\
\hline
\end{tabular}

Population size $(n)$ is given alongside the average percentage of missing genotype data, number of alleles $\left(n_{\mathrm{a}}\right)$, allelic richness $\left(a_{\mathrm{r}}\right)$, effective number of alleles $\left(n_{\mathrm{e}}\right)$, number of private alleles $\left(n_{\mathrm{p}}\right)$, observed heterozygosity $\left(H_{\mathrm{O}}\right)$, expected heterozygosity $\left(H_{\mathrm{E}}\right)$ and Wright's $F_{\mathrm{IS}}$ with significance indicated at the $5 \%$ level (*) and strict Bonferroni-corrected level $\left(\alpha=0.00028^{* *}\right)$

Table 6 Characterisation of 16 microsatellite loci for red-billed chough

\begin{tabular}{lllrlllrr}
\hline Locus name & Repeat unit & \multicolumn{1}{l}{$T_{\mathrm{a}}$} & $n_{\mathrm{a}}$ & Allele range & $H_{\mathrm{O}}$ & \multicolumn{1}{l}{$H_{\mathrm{E}}$} & $p_{\text {HWE }}$ & Null-allele frequency \\
\hline Ppy-001 & TACA & $60 \rightarrow 50$ & 4 & $151-179$ & $0.42 \pm 0.21$ & $0.41 \pm 0.19$ & 0.148 & $-0.04 \pm 0.13$ \\
Ppy-002 & ATCT & $60 \rightarrow 50$ & 4 & $151-179$ & $0.39 \pm 0.19$ & $0.39 \pm 0.20$ & 0.993 & $-0.05 \pm 0.12$ \\
Ppy-003 & AGAT & $60 \rightarrow 50$ & 11 & $292-344$ & $0.55 \pm 0.24$ & $0.55 \pm 0.15$ & $<0.001$ & $0.01 \pm 0.11$ \\
Ppy-004 & AGAT & $60 \rightarrow 50$ & 7 & $173-239$ & $0.45 \pm 0.22$ & $0.45 \pm 0.19$ & 0.183 & $-0.04 \pm 0.13$ \\
Ppy-005 & TATC & $60 \rightarrow 50$ & 7 & $226-250$ & $0.36 \pm 0.27$ & $0.36 \pm 0.26$ & 0.028 & $-0.02 \pm 0.14$ \\
Ppy-006 & CATC & $60 \rightarrow 50$ & 8 & $139-175$ & $0.13 \pm 0.30$ & $0.12 \pm 0.28$ & 0.729 & $0.00 \pm 0.05$ \\
Ppy-007 & GATA & $55 \rightarrow 45$ & 9 & $161-193$ & $0.58 \pm 0.16$ & $0.60 \pm 0.15$ & 0.425 & $0.00 \pm 0.08$ \\
Ppy-008 & GATA & $60 \rightarrow 50$ & 9 & $221-265$ & $0.57 \pm 0.14$ & $0.60 \pm 0.16$ & 0.018 & $-0.02 \pm 0.17$ \\
Ppy-009 & AAGT & $60 \rightarrow 50$ & 6 & $222-242$ & $0.56 \pm 0.25$ & $0.51 \pm 0.19$ & 0.420 & $-0.06 \pm 0.11$ \\
Ppy-010 & CA & $60 \rightarrow 50$ & 14 & $108-146$ & $0.50 \pm 0.26$ & $0.43 \pm 0.23$ & 0.187 & $-0.11 \pm 0.17$ \\
Ppy-011 & TAGA & $60 \rightarrow 50$ & 10 & $163-191$ & $0.68 \pm 0.16$ & $0.63 \pm 0.14$ & 0.190 & $-0.08 \pm 0.13$ \\
Ppy-012 & TAGA & $60 \rightarrow 50$ & 13 & $210-266$ & $0.47 \pm 0.23$ & $0.51 \pm 0.15$ & $<0.001$ & $0.00 \pm 0.23$ \\
Ppy-013 & GATA & $60 \rightarrow 50$ & 10 & $197-221$ & $0.64 \pm 0.12$ & $0.65 \pm 0.08$ & 0.493 & $0.01 \pm 0.07$ \\
Ppy-014 & GATG & $60 \rightarrow 50$ & 5 & $239-275$ & $0.39 \pm 0.11$ & $0.40 \pm 0.10$ & 0.615 & $0.02 \pm 0.08$ \\
Ppy-015 & TATG & $60 \rightarrow 50$ & 3 & $152-158$ & $0.13 \pm 0.26$ & $0.11 \pm 0.19$ & 0.120 & $-0.04 \pm 0.15$ \\
Ppy-016 & GGAT & $60 \rightarrow 50$ & 13 & $200-244$ & $0.53 \pm 0.23$ & $0.56 \pm 0.22$ & 0.022 & $0.02 \pm 0.07$
\end{tabular}

Statistics $( \pm 1 \mathrm{SD})$ were calculated from 348 individuals in 11 populations

The microsatellite repeat unit is given alongside TouchDown annealing temperature gradient $\left(T_{\mathrm{a}}\right)$, number of alleles $\left(n_{\mathrm{a}}\right)$, allele range $(b \mathrm{p})$, observed $\left(H_{\mathrm{O}}\right)$ and expected $\left(H_{\mathrm{E}}\right)$ heterozygosity, the probability of Hardy-Weinberg equilibrium $\left(p_{\mathrm{HWE}}\right)$ and null allele frequency (van Oosterhout et al. 2004). See Wenzel et al. (2011) for full characterisation

${ }^{a}$ Locus also isolated by Jaari et al. (2008)

\section{References}

Dávila JA, Morinha F, Blanco G (2014) Eleven new polymorphic microsatellite markers for the Red-billed chough (Pyrrhocorax pyrrhocorax). Conserv Genet Resour. doi:10.1007/s12686-014-0293-6

Excoffier L, Lischer HEL (2010) Arlequin suite ver 3.5: a new series of programs to perform population genetics analyses under Linux and Windows. Mol Ecol Resour 10:564-567

Jaari S, Välimäki K, Merilä J (2008) Isolation and characterization of 100 polymorphic microsatellite loci for the Siberian jay (Perisoreus infaustus). Mol Ecol Resour 8:1469-1474 van Oosterhout C, Hutchinson WF, Wills DP, Shipley P (2004) MICRO-CHECKER: software for identifying and correcting genotyping errors in microsatellite data. Mol Ecol Notes 4:535-538

Wenzel MA, Webster LMI, Segelbacher G, Reid JM, Piertney SB (2011) Isolation and characterisation of 17 microsatellite loci for the red-billed chough (Pyrrhocorax pyrrhocorax). Conserv Genet Res 3:737-740 